\title{
Prevalence of 1-Year Chronic Respiratory Morbidity Among Extremely Preterm \\ Infants and Related Hospital Length of Stay, Readmissions and Healthcare Costs: A Retrospective Claims Analysis
}

\section{Sujata P. Sarda, ${ }^{1}$ Alexandra Mangili, ${ }^{2}$ Linda Han, ${ }^{3}$ Rajeev Ayyagari, ${ }^{4}$ Wei Gao, ${ }^{4}$ Jessie Wang, ${ }^{4}$ Jing Zhao, ${ }^{4}$ Meredith Mowitz ${ }^{5}$ \\ ${ }^{1}$ Outcomes Research and Epidemiology (ORE), Shire, Lexington, MA, USA; ${ }^{2}$ Global Development, Shire, Zug, Switzerland; ${ }^{3}$ Global Clinical Development, Shire, Lexington, MA, USA; ${ }^{4}$ Analysis Group, Inc., Boston, MA, USA; ${ }^{5}$ Department of Pediatrics, College of Medicine, University of Florida, Gainesville, FL, USA

\section{BACKGROUND}

Chronic respiratory morbidity (CRM) is associated with extremely preterm birth. ${ }^{1}$

\section{OBJECTIVE}

To evaluate the prevalence of CRM, as well as the birth hospital length of stay (LOS), hospital readmission rates and healthcare costs for extremely preterm infants ( $\leq 28$ weeks gestational age [GA]) with and without CRM, through 1 year corrected age (CA)

\section{METHODS}

- This was a retrospective analysis of the Truven Health MarketScan claims database, which contains health

insurance claims and electronic medical records for patients in the United States.

- We included infants born at $\leq 28$ weeks GA who:

- Were admitted to a neonatal intensive care unit (NICU)

between January 2009 and June 2016

Survived to 36 weeks postmenstrual age

Had no congenital heart disease, diaphragmatic hernia or congenital malformations

- Had continuous insurance eligibility from birth to 1 year CA or death, whichever occurred first.

- CRM was defined as $\geq 1$ of the following events in $\geq 2$ quarters (3-month time intervals) through 1 year CA: hospital readmission or emergency room visit due to a respiratory cause, use of daily respiratory medication or use of home oxygen therapy/respiratory aids.

- Healthcare costs associated with birth hospitalisation outpatient visits, emergency room visits and hospital readmissions were evaluated for infants with CRM versus without CRM.

\section{RESULTS}

- The MarketScan database included 112,187 premature infants ( $<37$ weeks GA) born between January 2009 and June 2016 (Figure 1)

- Overall, 1782 eligible infants born at $\leq 28$ weeks were included in the current analyses.

1265 (71.0\%) did not have CRM, and 517 (29.0\%) had CRM.

- The proportion of female infants was higher in the cohort without versus with CRM (Table 1).

- A trend towards higher rates of comorbidities (bronchopulmonary dysplasia [BPD], intraventricular haemorrhage, retinopathy of prematurity, patent ductus arteriosus and necrotising enterocolitis) was observed among infants who experienced CRM (Table 1).

Approximately $86.7 \%$ of infants with CRM had BPD.

- Mean birth hospital LOS was significantly longer for infants with CRM versus those without (Table 2).

- Hospital readmission rates after NICU discharge, for all causes as well as for respiratory causes specifically, were significantly higher for infants with CRM versus those without (Table 2).

- Infants with CRM were more likely to have $>1$ hospital readmission, for all causes and respiratory causes, versus infants without CRM

- All healthcare costs were substantially higher for infants with CRM versus those without (Figure 2).

After the birth hospitalisation, which accounted for the highest costs, the largest drivers of increased costs were outpatient visits and hospital readmissions.
Figure 1. Sample Selection Flowchart of the Truven MarketScan Database

Premature infants $<37$ weeks GA born in hospital between January 2009 and June 2016 $n=112,187$

$$
\downarrow
$$

Infants admitted to ICU or NICU within 1 day after birth $\mathrm{n}=\mathbf{4 4 , 7 5 1}$

$$
\downarrow
$$

Infants who died before reaching 36 weeks PMA were excluded $\mathrm{n}=44,212$

$$
\downarrow
$$

Infants diagnosed with congenital heart disease, diaphragmatic hernia or congenital malformations were excluded $n=38,509$ $\downarrow$

Infants with continuous eligibility from birth to 3-month CA or death, whichever occurred first $n=24,076$

$$
\downarrow
$$

Infants $\leq 28$ weeks GA $\mathrm{n}=2805$

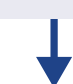

CRM/no CRM cohorts, $n=1782$ With CRM, $n=517(29 \%)$ Without CRM, $\mathrm{n}=1265(71 \%)$

Table 1. Patient Characteristics During Birth Hospitalisation

\begin{tabular}{|lcc|}
\hline Characteristic & $\begin{array}{c}\text { Without CRM } \\
\mathbf{n}=\mathbf{1 2 6 5}\end{array}$ & $\begin{array}{c}\text { With CRM } \\
\mathbf{n}=517\end{array}$ \\
\hline Female sex, $\mathrm{n}(\%)$ & $601(47.5)$ & $214(41.4)$ \\
GA, $\mathrm{n}(\%)^{\star}$ & & \\
$<24$ weeks & $37(2.9)$ & $39(7.5)$ \\
24 weeks & $102(8.1)$ & $98(19.0)$ \\
$25-26$ weeks & $404(31.9)$ & $212(41.0)$ \\
$27-28$ weeks & $722(57.1)$ & $168(32.5)$ \\
Multiple birth, $n(\%)$ & $342(27.0)$ & $130(25.1)$ \\
Delivery method, $n$ (\%) & & \\
Vaginal & $313(24.7)$ & $134(25.9)$ \\
Caesarean & $854(67.5)$ & $348(67.3)$ \\
Unknown & $98(7.7)$ & $35(6.8)$ \\
Comorbidities, $n$ (\%) & & \\
Bronchopulmonary dysplasia & $674(53.3)$ & $448(86.7)$ \\
Intraventricular haemorrhage & $334(26.4)$ & $193(37.3)$ \\
Retinopathy of prematurity & $403(31.9)$ & $229(44.3)$ \\
Patent ductus arteriosus & $595(47.0)$ & $322(62.3)$ \\
Necrotising enterocolitis & $114(9.0)$ & $68(13.2)$ \\
\hline
\end{tabular}

\section{CONCLUSIONS}

- The prevalence of CRM is considerable, as $29 \%$ of extremely preterm infants experience CRM through 1 year CA.

- CRM places a significant burden on healthcare systems.

- Compared with infants without CRM, infants with CRM have a longer birth hospital LOS, an increased rate of hospital readmission and increased healthcare costs.

REFERENCE

\begin{tabular}{|c|c|c|c|}
\hline & $\begin{array}{l}\text { Without CRM } \\
n=1265\end{array}$ & $\begin{array}{c}\text { With CRM } \\
n=517\end{array}$ & $\begin{array}{l}\text { Adjusted } \\
\text { PValue* }\end{array}$ \\
\hline \multicolumn{4}{|l|}{ Birth hospitalisation } \\
\hline $\begin{array}{l}\text { Mean } \pm \text { SD length } \\
\text { of stay, days }\end{array}$ & $78 \pm 31$ & $98 \pm 33$ & $<0.0001$ \\
\hline $\begin{array}{l}\text { Hospital readmission, } \\
\text { all-cause, } \mathrm{n}(\%)\end{array}$ & $180(14.2)$ & $209(40.4)$ & $<0.0001$ \\
\hline $\begin{array}{l}\text { No. of hospitalisations per } \\
\text { person-year } \\
\text { (incidence rate) }\end{array}$ & 0.18 & 0.64 & $<0.0001$ \\
\hline \multicolumn{4}{|l|}{$\begin{array}{l}\text { Length of stay among } \\
\text { infants with } \geq 1 \\
\text { hospital readmission }\end{array}$} \\
\hline Days per person-year & 7.53 & 10.40 & 0.0480 \\
\hline Median, days & 4.00 & 5.00 & - \\
\hline $\begin{array}{l}\text { Infants with }>1 \\
\text { readmission, } \mathrm{n}(\%)\end{array}$ & $32(2.5)$ & $69(13.3)$ & $<0.0001$ \\
\hline $\begin{array}{l}\text { Hospital readmission, } \\
\text { respiratory cause, } n(\%)\end{array}$ & $82(6.5)$ & $160(30.9)$ & $<0.0001$ \\
\hline $\begin{array}{l}\text { No. of hospitalisations per } \\
\text { person-year } \\
\text { (incidence rate) }\end{array}$ & 0.07 & 0.44 & $<0.0001$ \\
\hline \multicolumn{4}{|l|}{$\begin{array}{l}\text { Length of stay among } \\
\text { infants with } \geq 1 \\
\text { hospital readmission }\end{array}$} \\
\hline Days per person-year & 7.62 & 10.01 & 0.0877 \\
\hline Median, days & 4.00 & 5.00 & - \\
\hline $\begin{array}{l}\text { Infants with >1 } \\
\text { readmission, n (\%) }\end{array}$ & $3(0.2)$ & $48(9.3)$ & $<0.0001$ \\
\hline
\end{tabular}

Table 2. Length of Stay During Birth Hospitalisation, and Hospital Readmissions Within 1 Year After Birth Hospitalisation, in Extremely Preterm Infants With or Without CRM

Figure 2. Total Healthcare Costs* per Patient During 1 Yea CA Among Extremely Preterm Infants With or Without CRM CA Among Extreming Preth Birth Hospitalisation

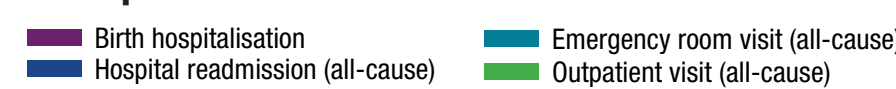

(A) $1,000,000 \quad P=0.0488^{\dagger}$
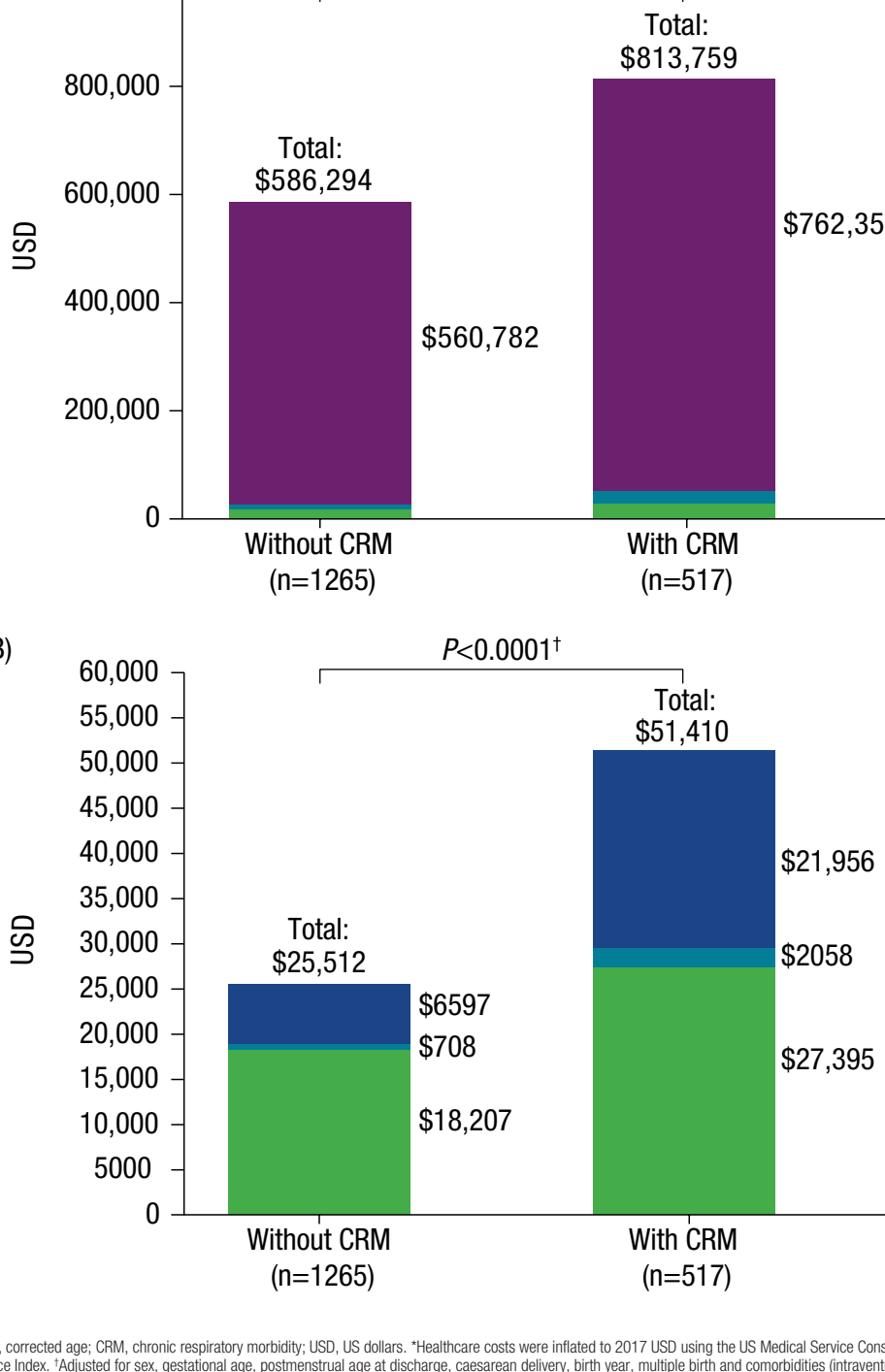

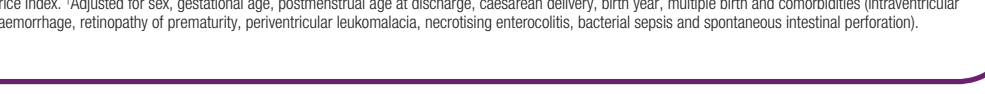

